\title{
POSSIBLE DENSITY DETERMINATION OF THE NEBULAR COMPONENT OF CATACLYSMIC VARIABLES WITH THE N III 4641/4635 LINE RATIO
}

\author{
Joaquín Bohigas, ${ }^{1}$ Eduardo Pérez-Tijerina,${ }^{2}$ and Roberto Machorro ${ }^{3}$
}

The dependence of the $N$ III $4641 / 4635$ line ratio on the electron density is presented. It is shown that this ratio may be an important diagnostic tool in cataclysmic variables.

Pérez-Tijerina, Bohigas \& Machorro (2001) used the Chianti code and database developed by the U.S. Nautical Observatory (Landi, 200) in order to find density and temperature sensitive line ratios in the conditions occuring in plasmas produced in pulsed laser deposition experiments for thin film deposition, i.c., around $10^{18} \mathrm{~cm}^{-3}$ and $10^{4} \mathrm{~K}$. Level populations are determined considering only radiative decay and collisional excitation and de-excitation. During this inquiry they found that the $\mathrm{N}^{+2} 4641 / 4635$ line ratio is density sensitive in threc quite different regimes (see Figure 1): $10-10^{4}, 10^{8}-10^{11}$ and $10^{16}-10^{20} \mathrm{~cm}^{-3}$.

This peculiar behavior is due to the interplay of the 20 levels included in this calculation. These permitted lines from $\mathrm{N}^{+2}$ have been observed in a variety of astrophysical objects, such as WR nebulae and cataclysmic variables. In order to test the utility of this line ratio as a density diagnostic tool for astrophysical plasmas, we used the spectral data produced by Mennicknet, Grenicr and Tovmassian (private communication 2003) for AE Cir, a cataclysmic variable candidate. From their spectrum we found that N III $1641 / 4635$ is between 0.73 and 1.08 (using the splot package in IRAF). From Figure 1 we find that this ratio would imply a density of $\sim 10^{3}, \sim 10^{10}$ or $\sim 10^{17} \mathrm{~cm}^{-3}$. The density must be larger than $10^{6} \mathrm{~cm}^{-3}$ since the $\mathrm{O}^{+2}$ lines at 4959 and $5007 \AA$ are absent. On the other hand it can not be close to $10^{17} \mathrm{~cm}^{-3}$ since this would imply a nebular mass in the vicinity of $1 M_{\odot}$, to large for these type of objects.

Therefore we conclude that, if the line ratio is indeed density sensitive in the physical environment of

\footnotetext{
${ }^{1}$ Instituto de Astronomía, l'N.MM, Apdo. Postal SiT. 22800 Ensenada, B.C., México (jbb@astrosen.unam.mx).

${ }^{2}$ Facultad de Ciencias Físico Matemáticas, INL. Apdo. Postal 101-F, 66450 San Nicolás de los Garza. N.L.. México (egperez@fcfm.uanl.mx).

${ }^{3}$ Centro de Ciencias de la Materia Condensacia. UN.M, Apdo. Postal 2681, 22800 Ensenada, B.C.. México (roberto@ ccmc.unam.mx).
}

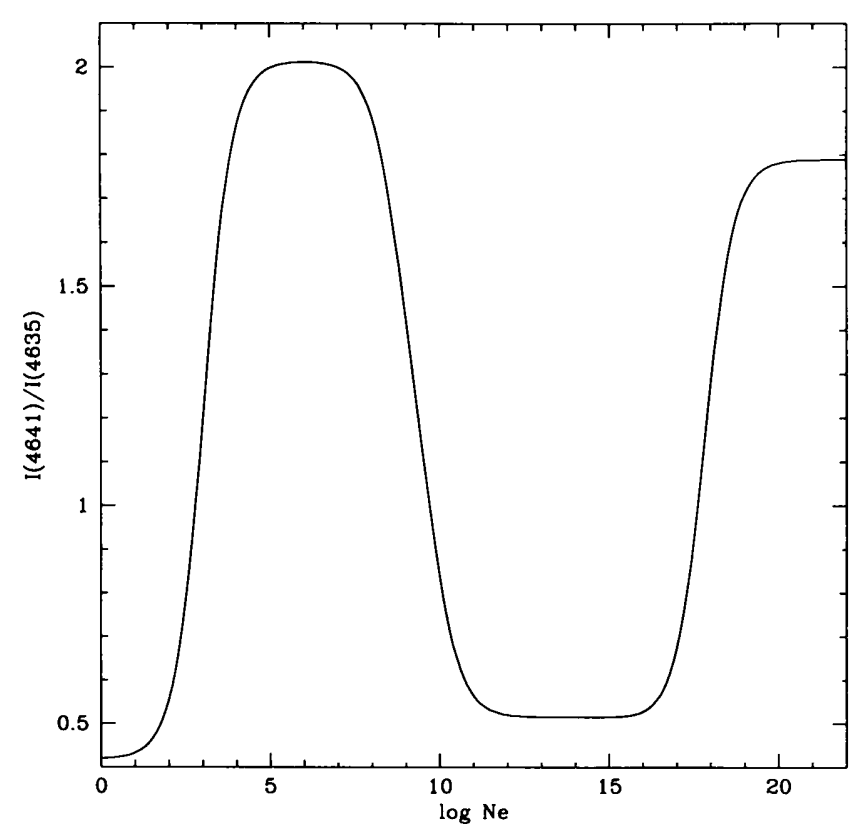

Fig. 1. N III $4641 / 4635$ as a function of the electron density for $T_{e}=10,000 \mathrm{~K}$

AE Cir, the nebular density must be between $4 \times 10^{9}$ and $2 \times 10^{10} \mathrm{~cm}^{-3}$, which implies that the nebular mass is close to $10^{-7} M_{\odot}$. These numbers are well within what is expected in cataclysmic variables.

This line ratio may be an important addition to the diagnostic tool kit used to inspect large density astrophysical nebulae. However, caution is advisable until other processes capable of populating the levels from which these two lines arise, such as as recombination, radiative excitation or the Bowen resonancefluorescence mechanism, are considered.

\section{REFERENCES}

Landi, E. 2000, RMexAA (Conf. Series) 9, 140

Pérez-Tijerina, E., Bohigas, J. \& Machorro, R. 2001, J. Appl. Phys. 90, 3192 